\title{
Sound taxation? On the use of self-declared value
}

\author{
Marco A. Haan* Pim Heijnen ${ }^{\dagger}$ Lambert Schoonbeek ${ }^{\ddagger}$ \\ Linda A. Toolsema ${ }^{\S}$
}

February 12, 2008

\begin{abstract}
In the 16th century, foreign ships passing through the Sound had to pay ad valorem taxes, known as the Sound Dues. To give skippers an incentive to declare the true value of their cargo, the Danish Crown reserved the right to purchase it at the declared value. We show that it is an equilibrium for the authorities to confiscate the cargo with some fixed probability independent of the declared value. This does not induce truth-telling, but does generate the desired tax revenue. Other applications of this framework include the dissolution of partnerships, and the auditing of income tax returns.
\end{abstract}

Keywords: Signaling; Taxation.

JEL classification: C72; H21; L33.

\footnotetext{
${ }^{*}$ Corresponding author. Department of Economics and Econometrics, University of Groningen, P.O. Box 800, 9700 AV Groningen, The Netherlands. m.a.haan@rug.nl.

${ }^{\dagger}$ CeNDEF, Department of Quantitative Economics, University of Amsterdam, Roetersstraat 11, 1018 WB, Amsterdam, The Netherlands. p.heijnen@uva.nl.

${ }^{\ddagger}$ Department of Economics and Econometrics, University of Groningen, P.O. Box 800, 9700 AV Groningen, The Netherlands. l.schoonbeek@rug.nl.

${ }^{\S}$ COELO, University of Groningen, P.O. Box 800, 9700 AV Groningen, The Netherlands. l.a.toolsema@rug.nl. The authors thank Tatiana Kiseleva for help in proving Proposition 3. Heijnen gratefully acknowledges financial support by the Netherlands Organization for Scientific Research (NWO).
} 


\section{Introduction}

In the 16th century, the Kingdom of Denmark controlled both sides of the Sound (Øresund), an important waterway at the time situated between present-day Denmark and Sweden. All foreign ships passing through this strait had to make a stop in Helsing $ø$ r (known in English as Elsinore, the stage for Shakespeare's Hamlet) and pay taxes to the Danish Crown, which amounted to $1-2 \%$ of the value of the cargo. These taxes are often referred to as Sound Dues. Although obviously unfamiliar with the concept of incentive compatibility, the Danish Crown was fully aware that such a tax would give skippers a strong incentive to cheat and declare a value much lower than the true one. It came up with an intriguing solution. The Crown reserved the right to purchase the cargo at the value declared by the skipper. Thus, a skipper who declared a value that was too low ran the risk of losing his cargo at a price below market value. But a skipper who declared a value that was too high ran the risk of paying too much in taxes. ${ }^{1}$

Although this mechanism is clearly ingenious, it does raise a number of questions. First, what is the optimal confiscation strategy for the tax authority? Obviously, it cannot be part of a Nash equilibrium to never purchase the cargo. Then, the threat of confiscation would simply be empty. Second, is this mechanism truth-revealing? That is, does it give skippers the incentive to always declare the true value of their cargo? Third, is this an optimal mechanism? Can it yield the desired tax revenue? Put differently, did this mechanism really amount to sound taxation or was there something rotten in the state of Denmark? We address these questions in this paper.

Obviously, the relevance of our analysis goes far beyond some tax scheme in Medieval Europe. There are numerous instances where a similar tax has either been proposed or implemented. In 1891, New Zealand passed a Land and Income Tax act, based on a "self-assessment with the shrewd device of

\footnotetext{
${ }^{1}$ This mechanism is described in some papers in the Economics literature (Gerchak and Fuller, 1992; Niou and Tan, 1994). It is also mentioned in Shasha (2007) and in Maczak (1972), as cited by Odlyzko (2004).
} 
making Government's purchase at the tax value an effective check on the owner's assessment" (Condliffe 1930, p. 182, as quoted by Niou and Tan, 1994). Dr. Sun Yat-sen, the first provisional president when the Republic of China was founded in 1912, proposed a land tax using the exact same mechanism: landowners are taxed according to their declared value of the land, and the government can also buy back the land at the same price. ${ }^{2}$ Anyone buying a house in southern Europe may face property taxes based on self-declared value. ${ }^{3}$ Other examples include land tax in India around 1900 as well as in present-day Taiwan, taxes on works of art leaving Mussolini's Italy, and British taxes on imported American Jerome clocks (see Gerchak and Fuller, 1992, and the references therein).

In our model we assume that, using the terminology of the Sound Dues example, the skipper knows the true value of his cargo, but the taxation authority does not. We thus analyze a signaling model with asymmetric information. We restrict our attention to cases in which the taxation authority has no clue about the true value. It could base its action on some estimate of the value, but we deliberately abstract from this possibility. We find that, in any equilibrium of our model, the tax authorities use a mixed strategy. Surprisingly, there are equilibria in which the probability that the authorities will confiscate the cargo does not depend on the declared value. In other words, there are equilibria in which the authorities use what we will refer

\footnotetext{
${ }^{2}$ Niou and Tan (1994) quote at length: "How indeed can the price of the land be determined? I would advocate that the landowner himself should fix the price. The landowner reports the value of his land to the government and the government levies a land tax accordingly... [T] he government makes two regulations: first, that it will collect taxes according to the declared value of the land; second, that it can also buy back the land at the same price... According to my plan, if the landowner makes a low assessment, he will be afraid lest the government buy back his land at that value and make him lose his property; if he makes too high an assessment, he will be afraid of the government taxes according to this value serious possibilities, he will certainly not want to report the value of his land too high or too low; he will strike a mean and report the true market price to the government" (Sun, 1924: 177-178).

${ }^{3}$ See the Guardian (2004): "In all three countries [France, Spain and Italy], these taxes are charged on the value of the property as declared in the deed of sale, which has traditionally borne little resemblance to the price actually paid. The penalties for tax dodging in this way are severe and include compulsory state purchase at the declared value, plus fines."
} 
to as a signal-independent strategy. If we restrict attention to such simple strategies, we can show that this tax scheme is not a truth-revealing mechanism, but that it does allow the taxation authorities to exactly collect the desired tax revenue in expected value. In a zero-sum game, any equilibrium has this property.

To derive these results, we develop a general model that applies to more than just taxation issues. We will show that a similar mechanism can also be used to dissolve a public-private partnership. Suppose that a public party wants to end some joint-venture with a private party. Also assume that the private party is fully informed about the true value of the joint-venture, but the public party is not. The public party can then ask the private party to name a price $m$, with the understanding that the public party could either decide to sell its own share to the private party at price $m$, or to buy the private party's share at price $m$. We use our general model to analyze this mechanism, and compare it to a case in which the public party makes a takeit-or-leave-it offer to the private party. We show that the first mechanism is desirable if the public party is sufficiently efficient in running the jointventure by itself.

In the case of income taxation, it is hard to imagine that a mechanism similar to that of the Sound Dues could be used. It is not feasible to acquire the entire income of a person at some price, especially if it is unclear how high that income actually is. Still, we will show that we can also apply our general model to such tax audits. Rather than the choice of whether or not to acquire some property, the tax authority then has the choice whether or not to audit some tax return. Qualitatively, the results are the same to those in the Sound Dues case: by randomly auditing a fixed fraction of tax returns, the tax authorities can make sure that they raise the desired tax revenue in expected value. Further applications of our framework include the use of shotgun clauses, and the allocation of an indivisible item.

We are not the first to give a game-theoretic treatment of these issues. Niou and Tan (1994) focus on Sun Yat-sen's land tax. They assume that the 
tax authority will use an audit to appraise the true value of the land before they decide whether to purchase it, and show that landowners will always underreport. In our model, we show that the tax authority does not need such appraisals. Also in our model landowners do underreport, but the tax authority is still able to obtain the desired tax revenue. Gerchak and Fuller (1992) focus on the dissolution of a partnership through a shotgun clause. They use a private-value set-up in which the declaring party is uncertain about the valuation of the claiming party. We focus on the case of a common value.

The remainder of this paper is structured as follows. In the next section we present our general model. That model is specified in general terms rather than being tailored to the Sound Dues application, to allow for alternative interpretations. In Section 3 we solve the general model, while we consider the special case of a zero-sum game in Section 3.2. Section 4 considers other applications of our framework, and presents some additional results. Section 5 concludes.

\section{The model}

We have two players, a declarer $d$ and a claimant $c$. We will refer to the declarer as being male, and to the claimant as being female. The timing of the game is as follows. First, Nature decides on the type $v$ of the declarer, which is his private information. It is common knowledge that $v$ is drawn from probability density function $f(v)$ with support $[0, \bar{v}]$. Second, the declarer announces some value $m$. For ease of exposition but without loss of generality, we assume that $m$ is restricted to some interval $[0, \bar{m}]$, where $\bar{m}$ can be arbitrarily large. Third, the claimant either accepts (A) or rejects (R) the message of the declarer. Both actions have well-defined consequences. In the Sound Dues example, the declarer is the skipper whose cargo has true value $v$. The value that he declares to the tax authorities is $m$. The claimant is the tax authority that can either accept the declaration, or reject it. If she accepts, the skipper pays a tax $t m$ which based on his announced value $m$, 
with $t \in(0,1)$. If she rejects, she buys the cargo at price $m$.

The payoffs in each outcome may depend both on the true type $v$ and on the signal $m$ of the declarer. Given $v$ and $m$, let $A_{d}(v, m)$ and $R_{d}(v, m)$ denote the payoff of the declarer in case the claimant chooses, respectively, Accept or Reject. Using similar notation, the payoffs to the claimant are denoted by $A_{c}(v, m)$ and $R_{c}(v, m)$. We assume that the payoff functions are differentiable in both arguments. In our Sound Dues example, if the claimant accepts, the declarer pays taxes $t m$, so payoffs are $A_{c}(v, m)=t m$ and $A_{d}(v, m)=-t m$. If she rejects, she buys the cargo at price $m$. We then have $R_{c}(v, m)=v-m$ and $R_{d}(v, m)=m-v$.

For our analysis, we need a number of mild assumptions on the payoff functions. First, we need the following separability assumption to hold:

Assumption 1. There exists a function $S(v)$ and a constant $c>0$, such that we can write $S(v)=A_{d}(v, m)+c \cdot R_{d}(v, m)$, for all $v$ and $m$.

In other words, we require that some linear combination of the payoffs to the declarer if the claimant accepts, and if she rejects, does not depend on the action $m$ of the declarer. Another way to interpret the assumption is that the "size of the cake" does not depend on the message $m$ that is sent - if we define the size of the cake as $A_{d}+c R_{d}$. More technically, this assumption implies that if the claimant always plays Accept with probability $1 /(1+c)$ and Reject with probability $c /(1+c)$, then the declarer is indifferent between all messages. The assumption is satisfied in the Sound Dues example, as $A_{d}(v, m)+t \cdot R_{d}(v, m)=-t m+t(m-v)=-t v$.

One direct implication of Assumption 1 is that ${ }^{4} \operatorname{sign}\left(\partial A_{d} / \partial m\right) \neq \operatorname{sign}$ $\left(\partial R_{d}\right) / \partial m$. We thus have two possibilities. First, if the claimant accepts, then the declarer benefits from sending a higher message, while if the claimant rejects, the declarer benefits from sending a lower message. Alternatively, it is exactly the other way round. We make a similar assumption for the payoffs to the claimant. Moreover, we assume that in cases that the declarer benefits

\footnotetext{
${ }^{4}$ We use the convention that $\operatorname{sign}(x)=+1$ if $x>0, \operatorname{sign}(x)=-1$ if $x<0$, and $\operatorname{sign}(x)=0$ if $x=0$.
} 
from a higher message, the claimant is hurt by a higher message, and viceversa. In that sense, there is always a conflict of interest between declarer and claimant. Taken together, these assumptions imply:

Assumption 2. Let $\operatorname{sign}\left(\frac{\partial R_{d}}{\partial m}\right)=\operatorname{sign}\left(\frac{\partial A_{c}}{\partial m}\right) \neq \operatorname{sign}\left(\frac{\partial R_{c}}{\partial m}\right)$ for all $v$ and $m$.

For a relevant problem, we require that the message $m$ affects the payoffs:

Assumption 3. Let $\frac{\partial R_{d}}{\partial m} \neq 0$ for all $v$ and $m$.

We also need that the incentives of the claimant depend on the type $v$ of the declarer. More precisely, we assume that the payoffs of the claimant are increasing in $v$ if she rejects, but decreasing in $v$ if she accepts - or vice-versa. Thus:

Assumption 4. Let $\operatorname{sign}\left(\frac{\partial R_{c}}{\partial v}\right) \neq \operatorname{sign}\left(\frac{\partial A_{c}}{\partial v}\right)$ for all $v$ and $m$.

This assumption also implies that, for each $m, R_{c}$ and $A_{c}$ cross at most once in $v$. Finally, we need the following single-crossing condition:

Assumption 5. For each $v \in[0, \bar{v}]$, there is a unique $m \in(0, \bar{m})$ such that $R_{c}(v, m)=A_{c}(v, m)$.

It is easy to see that all assumptions are satisfied for our Sound Dues example.

\section{Analysis}

In this section, we analyze the model that we set up above. We first analyze the general model in Section 3.1, focusing on Bayesian Nash equilibria that have a particularly simple form. Doing so allows us to derive the expected equilibrium payoffs to both players. In Section 3.2, we restrict attention to zero-sum games. For such games, we can derive expected payoffs in all Bayesian Nash equilibria. The implications for our Sound Dues example are discussed in Section 3.3. 


\subsection{General analysis}

A strategy for the declarer assigns to each type $v$ a probability density function $z_{v}(m)$, with support $[0, \bar{m}]$. Beliefs of the claimant assign to each message $m$ a probability distribution of possible types $\beta_{m}$ on $[0, \bar{v}]$. A strategy for the claimant is a function which, given beliefs $\beta_{m}$, maps $[0, \bar{m}]$ into the unit interval, i.e. $p:[0, \bar{m}] \rightarrow[0,1]$ where $p(m)$ denotes the probability with which the claimant plays Reject if she observes signal $m$. Both players want to maximize their ex ante expected payoffs.

We focus on equilibria that have a particularly simple form. Suppose that the claimant uses a strategy that does not depend on the message $m$ that the declarer sends. We will refer to such a strategy as a signal-independent strategy:

Definition 1. The claimant uses a signal-independent strategy if her action does not depend on the action of the declarer: $p\left(m_{i}\right)=p\left(m_{j}\right)$ for all $m_{i}, m_{j} \in[0, \bar{m}]$.

There are a number of reasons to focus on such strategies. First, since these strategies are simple, they require relatively little sophistication for the players in this game. Wilson (1987) criticizes most of the game theory literature as relying too much on common-knowledge assumptions, and argues for the use of simple mechanisms that do not require such assumptions. This is known as the Wilson Doctrine (see also e.g. Chung and Ely, 2007). Although our signal-independent strategies do not strictly satisfy Wilson's requirements, they are at least a step in that direction. Second, we will show that with signal-independent strategies, the tax authority is able to raise the desired tax revenue in expected value. In that sense, it cannot do better than using such strategies. It also implies that there is no necessity to second-guess the declarer. If the equilibrium yields the required tax revenue anyhow, then e.g. hiring an appraiser is completely redundant and would only add to the costs of tax collection. ${ }^{5}$ Third, there are obvious practical advantages to us-

\footnotetext{
${ }^{5}$ Note that this is something that Niou and Tan (1994) do worry about.
} 
ing a signal-independent strategy. The rule is easy to communicate to the agent in charge of the actual collection of taxes, and it is easy to monitor. The rule does not depend on the actual distribution of values, which implies it is stable through time and across circumstances.

We immediately have:

Proposition 1. Under Assumptions 1 to 5 it cannot be an equilibrium for the claimant to use a signal-independent strategy other than $p=p^{*} \equiv c /(1+c)$.

Proof. Suppose that the claimant uses some signal-independent strategy $p$. Take $p^{*}=c /(1+c)$. Using Assumption 1, the declarer is indifferent between all messages $m \in[\underline{m}, \bar{m}]$, i.e.

$$
\frac{\partial R_{d}}{\partial m} p^{*}+\frac{\partial A_{d}}{\partial m}\left(1-p^{*}\right)=0
$$

Suppose $p>p^{*}$. In that case, if we have $\partial R_{d} / \partial m>0$, then

$$
\frac{\partial R_{d}}{\partial m} p+\frac{\partial A_{d}}{\partial m}(1-p)>0
$$

which implies that all types $v$ of the declarer will choose $m=\bar{m}$. However, using $\partial R_{d} / \partial m>0$ and Assumptions 2 and 5 , we see that $R_{c}(v, \bar{m})<A_{c}(v, \bar{m})$ for all $v$, which implies that the claimant then wants to switch to Accept, i.e. set $p=0<p^{*}$. We thus obtain a contradiction. Similarly, if $\partial R_{d} / \partial m<0$, then given the strategy of the claimant, all types $v$ of declarer will play $m=0$. From $\partial R_{d} / \partial m<0$ and the Assumptions 2 and 5, we now know that $R_{c}(v, 0)<A_{c}(v, 0)$ for all $v$. Thus, again, we obtain a contradiction. A similar argument shows that the claimant cannot play some $p<p^{*}$.

Thus, if there is a Bayesian Nash equilibrium in which the claimant uses a signal-independent strategy, it must have $p(m)=p^{*}$ for all $m$. As we will illustrate in Section 4.2, there may be infinitely many signal-independent equilibria, as the declarer has great leeway in choosing his strategy. Two types of equilibria are of particular interest. In a separating equilibrium, each type of declarer chooses a different signal. Upon observing his signal, the claimant can thus infer the true type of the declarer. In a pooling equilibrium 
each type of declarer selects the same signal $m$, which makes $m$ completely uninformative. We can show the following:

Proposition 2. Under Assumptions 1 to 5 the game has exactly one fully separating equilibrium in which the claimant uses a signal-independent strategy, and exactly one pooling equilibrium in which the claimant uses a signalindependent strategy.

Proof. First note that in any equilibrium in which the claimant uses the strategy $p(m)=p^{*}$, she should be indifferent between Accept and Reject. This implies the following restriction:

$$
\mathbb{E}\left[R_{c}(v, m) \mid m\right]=\mathbb{E}\left[A_{c}(v, m) \mid m\right] \Leftrightarrow \mathbb{E}[g(v, m) \mid m]=0,
$$

where $g(v, m) \equiv R_{c}(v, m)-A_{c}(v, m)$. The conditional distribution of $v$ given $m$ follows from the strategy of the declarer.

We know from Assumption 5 that for each type $v$ of declarer there exists a unique $m, m^{*}(v)$ say, such that $g\left(v, m^{*}(v)\right)=0$. Using the implicit function theorem and Assumptions 2, 3 and 4 we see that $\frac{\mathrm{d} m^{*}}{\mathrm{~d} v}=\frac{\partial g / \partial m}{\partial g / \partial v}$ is either strictly positive or strictly negative. As a consequence $m^{*}(v)$ is strictly monotonic. Observing that $\mathbb{E}[g(v, m) \mid m]=g\left(v, m^{*}(v)\right)=0$, it easily follows that we have a unique separating equilibrium in which the declarer plays strategy $m^{*}(v)$ and the claimant plays Reject with probability $p^{*}$. In this equilibrium, the conditional distribution of $v$ given $m$ is very simple, since $v$ is known with certainty given $m$.

Next, consider a pooling equilibrium in which each type $v$ of declarer selects the same signal. Define the continuous function $H(m) \equiv \mathbb{E}[g(v, m) \mid m]=$ $\int_{0}^{\bar{v}} g(v, m) f(v) \mathrm{d} v$. In a pooling equilibrium the signal $m$ chosen by the declarer must be such that $H(m)=0$. Note that

$$
\frac{\partial H}{\partial m}=\int_{0}^{\bar{v}} \frac{\partial g(v, m)}{\partial m} f(v) \mathrm{d} v .
$$

Suppose now that $\partial R_{d} / \partial m>0$. Then $\partial g / \partial m<0$ from Assumption 2, and thus $\partial H / \partial m<0$. Moreover, using Assumption 5 we then know that for 
all $v$ we have $g(v, 0)>0$ and $g(v, \bar{m})<0$. In turn, this implies that $H(0)>0$ and $H(\bar{m})<0$. Hence, we see that there exists a unique signal, $m^{* *}$ say, such that $H\left(m^{* *}\right)=0$. It easily follows that we have a pooling equilibrium in which all types of declarer give the signal $m^{* *}$ and the claimant plays Reject with probability $p^{*}$. The case with $\partial R_{d} / \partial m<0$ can be discussed in a similar way. Finally, note that in the pooling equilibrium the conditional distribution of $v$ given $m$ is simply equal to the unconditional distribution of $v$.

The proofs of the propositions above also suggest how we can construct equilibria with signal-independent strategies. We have from the proof of Proposition 1 that, in such an equilibrium, the declarer is necessary indifferent between all possible messages he can send. For any $v$, there always exists a unique $m^{*}(v)$ that makes the claimant indifferent between accepting and rejecting. The unique fully separating equilibrium then has a declarer of type $v$ sending exactly the message $m^{*}(v)$. Upon observing $m$, the claimant then exactly knows which type of declarer she is facing, which is $v(m)=m^{*-1}(m)$.

But more equilibria exist. For the claimant to be willing to mix between Accept and Reject, it is not necessary that she can exactly infer the type of declarer she is facing from observing the message $m$ that the declarer sends. It is sufficient that she faces the required type $v$ on average. Consider for example the situation in Figure 1. Suppose that types are uniformly distributed. Suppose that, for this particular situation, the function $m^{*}(v)$ is given by the straight line. Then it is part of the fully separating equilibrium for the declarer to always exactly play $m^{*}(v)$. But the declarer may just as well add some noise to his signal. Suppose that a type $v$ plays a message $m$ that is drawn from a uniform distribution between the two dotted curves. From the figure, it is then easy to see that, for the claimant, the expected value of $v$ given $m$ is not affected. Hence, this strategy is also part of an equilibrium.

Our unique pooling equilibrium takes this argument to the extreme. It can even be an equilibrium for the declarer to play the same message $m^{* *}$, regardless of his type - as long, given the distribution of types, $m^{* *}$ is con- 


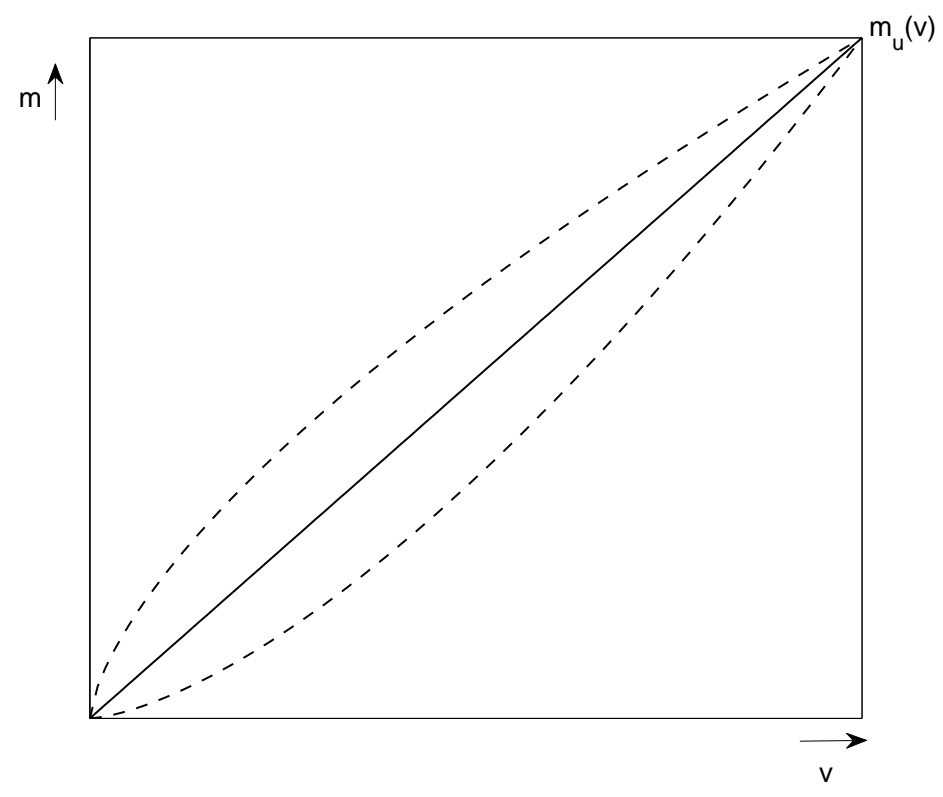

Figure 1: Equilibria

structed in such way that the claimant is exactly indifferent between Accept and Reject.

From the discussion above, we immediately have that the declarer has the same expected payoff in any signal-independent equilibrium. The expected payoff of the claimant seems to depend on the strategy used by the declarer. In case $R_{c}(v, m)$ and $A_{c}(v, m)$ are linear in both $m$ and $v$, however, the expected payoff of the claimant is independent of the declarer's strategy.

Proposition 3. If $R_{c}(v, m)$ and $A_{c}(v, m)$ are linear in both $v$ and $m$, and Assumptions 1-5 are satisfied, then each Bayesian Nash equilibrium in which the claimant uses a signal-independent strategy yields the same expected payoff for the declarer and the claimant. The expected payoff for the declarer is $\frac{1}{1+c} \mathbb{E}[S(v)]$ and the expected payoff for the claimant is proportional to $\mathbb{E}[v]$.

Proof. In Appendix. 


\subsection{The zero-sum game}

Now consider the zero-sum version of our game, where $R_{d}(v, m)+R_{c}(v, m)=$ 0 and $A_{d}(v, m)+A_{c}(v, m)=0$ for all $v$ and $m$. Hence, this reflects situations in which the declarer makes (or receives) the payment directly to the claimant. This is also satisfied for the Sound Dues example. Note however that this does not imply that our game is a classic two-person simultaneousmove complete-information zero-sum game: despite that payoffs always sum to zero, we still have a signalling model with asymmetric information.

Under this assumption, we can show that every equilibrium of our game (and not just the equilibria in which the claimant plays a signal-independent strategy) has the same ex ante expected payoffs for both players. Luce and Raiffa (1957, Appendix 2) show that a characteristic of complete-information zero-sum games is that any equilibrium has the same ex ante equilibrium payoffs. We can show that the same result holds in our zero-sum game with incomplete information. In turn, this implies for our Sound Dues example that the claimant is always able to achieve the desired tax revenue.

We first establish the following result.

Proposition 4. Any equilibrium of the zero-sum game necessarily has the claimant being indifferent between Accept and Reject, for every message $m$.

Proof. First note that the result trivially holds for those $m$ in which the claimant strictly mixes between the two actions. Next, let $p(m)=1$ for some $m$ and let $v$ be a type of declarer that selects this signal $m$ with positive probability in equilibrium. Note that, given the signal $m$, the claimant plays Reject with certainty. We will argue now that for the given $v$ and $m$ we must have $g(v, m) \leq 0$. Suppose otherwise that $g(v, m)>0$. There are two cases to distinguish: $\partial R_{d} / \partial m>0$ and $\partial R_{d} / \partial m<0$. The proof is similar for the two cases and shown only for $\partial R_{d} / \partial m>0$. Observe that in case $\partial R_{d} / \partial m>0$ we can find an $m^{\prime}>m$ and $m^{\prime}$ sufficiently close to $m$ such that both $A_{d}\left(v, m^{\prime}\right)>R_{d}(v, m)$ and $R_{d}\left(v, m^{\prime}\right)>R_{d}(v, m)$. Hence, regardless of the value of $p\left(m^{\prime}\right)$, it is always optimal for a declarer of type $v$ to deviate 
to $m^{\prime}$, which cannot be possible in equilibrium. Therefore, $p(m)=1$ implies that $g(v, m) \leq 0$ for all types $v$ of declarer that play action $m$ in equilibrium.

Furthermore, in the case that $p(m)=1$, the claimant's payoff from playing Reject should be at least as large as the payoff from playing Accept: $\mathbb{E}\left[R_{c}(v, m) \mid m\right] \geq \mathbb{E}\left[A_{c}(v, m) \mid m\right]$, or equivalently, $\mathbb{E}[g(v, m) \mid m] \geq 0$. This contradicts with $g(v, m) \leq 0$ unless $\mathbb{E}[g(v, m) \mid m]=0$.

The proof for the case that $p(m)=0$ for some $m$ goes along the same lines.

We then have:

Proposition 5. Under Assumptions 1 to 5, the zero-sum game has the same ex ante expected payoffs in any equilibrium: $\frac{1}{1+c} \mathbb{E}[S(v)]$ for the declarer and $-\frac{1}{1+c} \mathbb{E}[S(v)]$ for the claimant.

Proof. By Proposition 4, in any equilibrium the claimant is indifferent between Reject and Accept. In particular, $\mathbb{E}\left[R_{c}(v, m) \mid m\right]=\mathbb{E}\left[A_{c}(v, m) \mid m\right]$. Using $R_{d}(v, m)=-R_{c}(v, m)$ and Assumption 1, we then have:

$$
\mathbb{E}\left[R_{d}(v, m) \mid m\right]=\mathbb{E}\left[S(v)-c \cdot R_{d}(v, m) \mid m\right]
$$

or

$$
\mathbb{E}\left[R_{d}(v, m) \mid m\right]=\frac{1}{1+c} \mathbb{E}[S(v) \mid m] .
$$

The ex ante expected payoff in equilibrium is the expected value over $m$. For the declarer, this is

$$
\mathbb{E}\left[\mathbb{E}\left[R_{d}(v, m) \mid m\right]\right]=\frac{1}{1+c} \mathbb{E}[S(v)] .
$$

The result for the claimant follows directly.

Hence, the ex ante expected payoff of each player is a function of only $S(\cdot), c$ and the distribution of $v$, and can be calculated without knowing the precise equilibrium strategies of both players. In particular, in the context of zero-sum games this is also true if the claimant does not restrict attention to signal-independent strategies. 


\subsection{Sound Dues}

The above results have important implications for our Sound Dues example, which is a zero-sum game with payoffs $R_{d}(v, m)=-(v-m)=-R_{c}(v, m)$ and $A_{d}(v, m)=-t m=-A_{c}(v, m)$. Note that $S(v)=-t v$ and $c=t$. From Proposition 5 we immediately have that in any equilibrium the ex ante expected tax revenue equals $\frac{t}{1+t} \mathbb{E}[v]$. With complete information the tax revenue would be $t \mathbb{E}[v]$. Hence, in any equilibrium the taxpayer on average underreports his valuation by a factor $\frac{1}{1+t}$. At this point the expected cost of receiving a lower price for the asset in case of purchase is equal to the expected benefit of paying a lower tax. The government can use this knowledge to adjust the tax rate and raise precisely the desired amount of tax revenue that would be raised if $v$ were complete information.

Proposition 6. In the Sound Dues game, the King of Denmark can achieve any desired effective tax rate $t^{*} \in[0,1 / 2)$ in expected value by imposing a true tax rate $t=t^{*} /\left(1-t^{*}\right)$, and by requiring the toller to confiscate cargo with fixed probability $p^{*}=t /(1+t)$, regardless of its declared value.

This proposition shows that the taxation scheme discussed above is efficient in the following sense. Suppose that the King has decided that the optimal tax rate is some $t^{*}$. How this optimal tax rate has been determined, is immaterial and beyond the scope of this paper. It could be the tax rate that maximizes expected tax revenue, but the King could also have some other objective function. Then the proposition implies that the King can exactly raise the desired tax revenue $t^{*} v$ in expected value by imposing a tax rate $t=t^{*} /\left(1-t^{*}\right)$ and playing a signal-independent strategy. Note that this is true even though the taxpayer does not declare the true value in equilibrium. In the separating equilibrium of Proposition 2, we even have that each individual precisely pays the desired tax burden, rather than only in expected value. The Sound toll scheme is a sound taxation scheme. Note however that the mechanism is not truth-telling, and in most cases not even 
truth-revealing. ${ }^{6}$ But for the expected tax revenue, this is immaterial.

In the analysis above, we assumed that any given cargo is worth just as much to the skipper as it is to the tax authority. In practice, this may not be true. It is likely that the tax authority faces some transaction costs from appropriating a cargo and selling it on the market. Our framework easily allows for that possibility. Suppose for example that a cargo that is worth $v$ to the skipper only has value $\phi v$ for the tax authority, with $\phi \in$ $(0,1)$. In that case, we no longer have a zero-sum game. Still, we can use the analysis in Section 3.1 to construct equilibria with signal-independent strategies. Consider for example a fully separating equilibrium. We still have $A_{d}(v, m)=-t m, A_{c}(v, m)=t m, R_{d}(v, m)=-(v-m)$, but $R_{c}(v, m)=\phi v-$ $m$. The value of $c$ in Assumption 1 still is $t /(1+t)$, but for the tax authority to be indifferent between Accept and Reject, we now need $m=\phi v /(1+t)$. The expected revenue for the tax authority then is $\frac{\phi t}{1+t} \mathbb{E}[v]$ in any signalindependent equilibrium. Again, this implies that the King can achieve any effective tax rate, provided $\phi$ is not too high. Different from the zero-sum case however, we no longer have that this is true in any equilibrium: it only holds in the signal-independent equilibria.

\section{Other applications}

In the previous sections, we set up a general model and applied it to the Sound Dues case. In this section, we discuss some further applications of our general framework. Table 1 presents payoffs for the general model as well as for the applications that we discuss. In all applications we select some arbitrarily large values for $\bar{m}$ and $\bar{v}$. We focus on the fully separating equilibrium discussed in Proposition 2 and/or the ex ante equilibrium payoffs.

\section{[INSERT TABLE 1 ABOUT HERE]}

\footnotetext{
${ }^{6}$ It only is in the separating equilibrium described in Proposition 2.
} 


\subsection{Dissolving a public-private partnership}

In this subsection we apply our framework to the case of terminating a publicprivate partnership. Such partnerships, in which a private party and a public party both own half of the project, are often used in e.g. infrastructure projects. At some point the public party may consider to end the partnership, either by transferring control fully to the private party, or by obtaining full control for itself. The problem is that the private party is often better informed about the value of the business. The public party could then use a mechanism not unlike that in the Sound Dues example: it could ask the private party to name a price $m$ with the understanding that the public party could either decide to sell its own share to the private party at price $m$, or to buy the private party's share at price $m$. Alternatively, the private party could itself announce a price $k$ at which it is willing to either sell its own share, or to buy the share of the private party. An obvious question is which mechanism would yield the highest expected revenue for the private party. We will show that the private party wants to name a price itself if it is sufficiently inefficient in running the project.

A private party owns half of the shares in some project, the government owns the other half. The private party manages the project and has inside information about the true value $v \geq 0$. The government wants to end the partnership. It invites the private party to announce the price $m \geq 0$ at which it is willing to sell its share in the project. However, the government also reserves the right to sell its share to the private party at that price.

We assume that the government may be less efficient than the private party in running the firm, i.e. we assume that the true value to the government is $\delta(v, r)$, where the parameter $r \in[0,1]$ is a measure of government efficiency, and $\delta(v, 0)=0$ for all $v, \delta(v, 1)=v$ for all $v$, and $\partial \delta(v, r) / \partial r>0$ for all $v$ and $r$. Note that $0 \leq \delta(v, r) \leq v$ for all $v$ and $r$. The value of $r$ is common knowledge.

This game corresponds to the one discussed in Section 2 with the declarer the private party and the claimant the government. The action Reject corre- 
sponds to privatization (selling the government's stake), while action Accept corresponds to nationalization (buying the stake of the private party). If the government rejects, the private party obtains an additional half of the project at a price $m$, while the true value is $v$. The governments sells its share at price $m$. Hence $R_{d}(v, m)=v-m$ and $R_{c}(v, m)=m$. If the government accepts, it buys the private party's share at a price $m$, so $A_{d}(v, m)=m$ and $A_{c}(v, m)=\delta(v, r)-m$. Moreover $S(v)=v$ and $c=1$ (see Table 1). Note that we set the value of the original $50 \%$ share equal to zero, implicitly assuming that retaining only a $50 \%$ share is not an option. ${ }^{7}$

For the sake of argument, focus on the fully separating equilibrium described in Proposition 2. In this equilibrium we have $m^{*}(v)=\frac{1}{2} \delta(v, r)$ and $p^{*}=\frac{1}{2}$. The corresponding ex ante expected revenue is $\frac{1}{2} \mathbb{E}[\delta(v, r)]$ for the government and $\frac{1}{2} \mathbb{E}[v]$ for the private party, so both obtain half of what the project is worth if they run it themselves. From Proposition 3, we have that all other signal-independent equilibria yield the same expected revenues in the case that $\delta(v, r)$ is linear in $v$. Of course, if $r<1$, this is not an efficient outcome since joint payoffs are then maximized when the government sells its shares with probability 1.

An alternative mechanism for the government is to announce a price $k \geq 0$, rather than inviting the informed private party to do so. Given $r$, the government maximizes

$$
\begin{aligned}
\Delta(k, r) & =k[1-F(k)]+\mathbb{E}[\delta(v, r)-k \mid v \leq k] \\
& =k[1-2 F(k)]+\int_{0}^{k} \delta(v, r) f(v) \mathrm{d} v,
\end{aligned}
$$

with $F(\cdot)$ the cumulative distribution function of $v$. Assume that $\Delta(\cdot)$ has a unique interior maximum, and solve the first-order condition (FOC)

$$
1-2 F(k)+(\delta(k, r)-2 k) f(k)=0 .
$$

\footnotetext{
${ }^{7}$ Alternatively, we might assume that the value of the original $50 \%$ share is $\frac{1}{2} \gamma(v)$ to either party, where $0 \leq \gamma(v) \leq v$. To obtain the 'additional' payoffs of the game, we now need to subtract $\frac{1}{2} \gamma(v)$ from each from the payoffs $R_{d}(v, m), R_{c}(v, m), A_{d}(v, m)$ and $A_{c}(v, m)$. Thus, we may ignore the term $\frac{1}{2} \gamma(v)$.
} 
As $\delta(k, r)-2 k<0$, we have that at the optimal solution $F(k)<\frac{1}{2}$, so the optimal price $\hat{k}(r)$ is smaller than the median of the valuation distribution.

Comparing $\frac{1}{2} \mathbb{E}[\delta(v, r)]$ with $\Delta(\hat{k}, r)$, we have

Proposition 7. There is an $r^{\prime} \in(0,1]$ such that the government has a higher ex ante expected revenue by announcing a price itself if $r<r^{\prime}$, and by inviting the private party to announce a price if $r \geq r^{\prime}$.

Proof. First, take $r=0$. We then show that $\Delta(\hat{k}(0), 0)>\frac{1}{2} \mathbb{E}[\delta(v, 0)]$. Note that

$$
\Delta(k, 0)=k[1-2 F(k)] .
$$

Thus, $\Delta(\hat{k}(0), 0) \leq 0$ implies that $1-2 F(\hat{k}(0)) \leq 0$, which violates the FOC (7). So, we must have $\Delta(\hat{k}(0), 0)>0=\frac{1}{2} \mathbb{E}[\delta(v, 0)]$.

Second, take $r=1$. We then show that $\Delta(\hat{k}(1), 1) \leq \frac{1}{2} \mathbb{E}[\delta(v, 1)]=\frac{1}{2} \mathbb{E}[v]$. Consider therefore the situation in which the government announces the price $k$. Suppose, for the moment, that $v$ were known to the government. In that case we have the following: (i) if $v-k>k$, then the project is privatized and the government's revenue is $k$, and (ii) if $v-k \leq 0$, then the project is fully nationalized and the government's revenue is $v-k$. Hence, if $v$ were known to the government, its revenue would be $v-\max \{v-k, k\}$. This expression is maximized if $k=\frac{1}{2} v$, so with complete information the ex ante expected revenue of the government would be $\frac{1}{2} \mathbb{E}[v]$. Return now to the case in which the true $v$ is unknown to the government. Then, the government's revenue is at most $\frac{1}{2} \mathbb{E}[v]$, since it cannot possibly be worse off if it has more information.

The above analysis implies that $\Delta(\hat{k}(r), r)$ and $\frac{1}{2} \mathbb{E}[\delta(v, r)]$ as a function of $r$ cross an odd number of times. In order to show that they cross exactly once, notice by using the envelope theorem that

$$
\frac{\mathrm{d} \Delta(\hat{k}(r), r)}{\mathrm{d} r}=\int_{0}^{\hat{k}(r)} \frac{\partial \delta}{\partial r} f(v) \mathrm{d} v>0,
$$

and

$$
\frac{\mathrm{d} \frac{1}{2} \mathbb{E}[\delta(v, r)]}{\mathrm{d} r}=\frac{1}{2} \int_{0}^{\bar{v}} \frac{\partial \delta}{\partial r} f(v) \mathrm{d} v>0 .
$$


Since $\hat{k}(r)$ is increasing in $r,{ }^{8}$ the derivatives intersect at most once. But if $\Delta(\hat{k}(r), r)$ and $\frac{1}{2} \mathbb{E}[\delta(v, r)]$ cross $n$ times, the derivatives must intersect at least $n-1$ times. Hence, there are at most two intersections of $\Delta(\hat{k}(r), r)$ and $\frac{1}{2} \mathbb{E}[\delta(v, r)]$. Since there is an odd number of intersections, there is only one intersection.

Hence, only a relatively efficient government finds it optimal to invite the private party to announce a price at which the government will either sell or buy. An inefficient government is better off announcing a price itself. The intuition is as follows. By letting the private party announce a price, the public party gives away its bargaining power, but can keep the private party in check by the threat of buying at the price that the private party chooses. But as the public party becomes more inefficient, this becomes less of a threat, as the private party knows that the public party is not able to get much value out of the entire firm, and hence is also not willing to pay a high price.

\subsection{Shotgun clauses}

In this subsection we analyze the dissolution of a partnership via a buysell (or put-call) clause. Such clauses, also known as Shotgun clauses, are often included in partnership agreements. The party that wishes to end the relationship (the declarer) gives his estimate of the value of the firm, the other party (the claimant) then has the choice of buying his share, or to sell her share to him at the declared price (see Gerchak and Fuller, 1992).

For our framework to apply, we do need that the declarer has private information about the true value of $v$, while the claimant does not have that information. This is the case, for example, if the declarer is an insider to the business, say an owner-manager, and the responder is an outside owner. We assume that the claimant owns a fraction $s$ of the business initially, where $0<s<1$.

\footnotetext{
${ }^{8}$ This can be verified by totally differentiating the FOC (7) and using the second-order condition.
} 
The declarer announces a value $m \geq 0$, after which the claimant may either sell her share at the declared value (Reject) or buy him out at this price (Accept). ${ }^{9}$ We thus have a zero-sum game. Also note that this application is closely related to the one in the previous section. We use this application to illustrate that there may be infinitely many signal-independent equilibria.

The payoffs of this zero-sum game are given by $R_{d}(v, m)=(v-m) s=$ $-R_{c}(v, m)$ and $A_{d}(v, m)=-(v-m)(1-s)=-A_{c}(v, m)$ (see Table 1$)$, which satisfy the assumptions of our general model, with $S(v)=0$ and $c=(1-s) / s$.

We immediately have that the expected equilibrium payoff as given in Proposition 5 equals zero. This implies that on average the declared value is equal to the true value: $\mathbb{E}[m]=\mathbb{E}[v]{ }^{10}$ Using Proposition 2 we find the fully separating equilibrium where the declarer announces the true value, i.e. $m^{*}(v)=v$, and the responder sells with probability $p^{*}=1-s$. But as we mentioned before, the declarer has considerable leeway in constructing his strategy. If the responder uses $p^{*}=1-s$, then any strategy for which $\mathbb{E}[g(v, m) \mid m]=0$ will do. Here the latter reduces to

$$
\mathbb{E}[-(v-m) s-(v-m)(1-s) \mid m]=0 \Leftrightarrow \mathbb{E}[v \mid m]=m
$$

Hence, for example, the following also constitutes an equilibrium strategy for the declarer: set $m$ equal to $\mathbb{E}[v]+\epsilon$ with probability $\frac{1}{2}$ and equal to $\mathbb{E}[v]-\epsilon$ otherwise, where $\epsilon>0$. Note that there are infinitely many strategies of this form.

\subsection{Tax auditing}

Consider the case of income tax auditing. Suppose that a citizen has income $v \geq 0$, and is obliged to pay taxes at a rate $t$. Suppose that he reports an income of $m \geq 0$. If the tax authority accepts this declaration, he faces a total tax bill of $t m$, thus $A_{d}(v, m)=-t m$ and $A_{c}(v, m)=t m$. However,

\footnotetext{
${ }^{9}$ Different from Gerchak and Fuller (1992), we assume that the value $v$ is the true value to both players, and that the responder does not know $v$.

${ }^{10}$ Gerchak and Fuller (1992) conclude instead that the declarer will always underreport the value of the business.
} 
the tax authority may also choose to audit this tax return. The costs of doing so are $K>0$. By doing an audit, the tax authority learns true income $v$, taxes this income at a rate $t$, and also imposes a fine at a rate $f>t$ on the difference between true income $v$ and declared income $m$. Hence $R_{d}(v, m)=-t v-f(v-m)$ and $R_{c}(v, m)=t v+f(v-m)-K{ }^{11}$

To find a fully separating equilibrium, we first, we need to show that it is possible to find a $c$ such that the sum $A_{d}(v, m)+c \cdot R_{d}(v, m)$ does not depend on $m$. It is easy to see that this is true for $c=t / f$. Next, we need a reporting strategy $m(v)$ that makes the tax authority always indifferent between Accept and Reject. Hence we need $m(v)$ such that

$$
t m(v)=t v+f v-f m(v)-K,
$$

which implies ${ }^{12} m^{*}(v)=v-K /(t+f)$.

Hence, as in the Sound Dues example, the tax authority is able to obtain the desired tax revenue by simply using a signal-independent strategy. That is, the tax authority will audit a fixed fraction of tax returns, but will pick those returns at random, without taking into account the income that is declared on those returns. In the separating equilibrium, all citizens declare an income that is too low. The income that is declared, is decreasing in the costs of an audit $K$, but increasing in the tax rate $t$ and the fine $f$.

\subsection{Allocating an indivisible item}

As a final example, consider a situation where two players are to allocate an indivisible item with value $v \geq 0$ among themselves. They both own an equal share but only player 1 (the declarer) knows the true value. One way of doing this is by a 'divide-and-choose' method (see e.g. Moldovanu, 2002). Both players deposit a large sum $T / 2(T>>v)$ into a pot also including the

\footnotetext{
${ }^{11}$ Note that this specification implicitly assumes that the citizen will always choose to set $m \leq v$ : with $m>v$ the specification would imply a bonus for overreporting one's income. Since the equilibrium that we will find does have $m<v$, we ignore this problem.

${ }^{12}$ For this to be an equilibrium, we need $m^{*}(v)>0 \forall v$, which implies that we need a lower bound of $\underline{v} \geq K /(t+f)$ for the possible values of $v$. Having such a lower bound does not affect our results.
} 
item. The declarer then splits the pot into two parts, one of which contains the item plus an amount $m \geq 0$, after which the claimant may choose his preferred part - either the part including the item (which corresponds to the action Reject) or the part with money only (Accept).

Again we have a zero-sum game with $R_{d}(v, m)=-\frac{1}{2}(v-T)-m=$ $-R_{c}(v, m)=-A_{d}(v, m)=A_{c}(v, m), S(v)=0$ and $c=1$ (see Table 1), which satisfy the assumptions of our general model. From Proposition 5 we immediately have that the ex ante expected payoffs to both players are zero, which indicates that this method for dividing the indivisible item is fair. In the fully separating equilibrium of Proposition 2 we have $m^{*}(v)=\frac{1}{2} T-\frac{1}{2} v$ and $p^{*}=\frac{1}{2}$. The main difference between this game and the previous games is that in the separating equilibrium the value $m$ now depends negatively on the true value $v$.

\section{Conclusion}

In this paper, we studied an ingenious method used by Danish authorities in Medieval times to collect taxes. The authorities raised taxes on the basis of the declared value of the cargo, but reserved the right to purchase the cargo at that declared value. We showed that, although this is not a truthrevealing mechanism, it does allow the authorities to raise the desired tax revenue in expected value. This is always true in zero-sum games, but also in non-zero-sum games if we restrict attention to signal-independent strategies for the Danish authorities.

Apart from raising the required tax revenue, the mechanism has other obvious advantages. It is simple, and therefore easy to communicate to any civil servant that is involved in the collection of taxes. It is relatively cheap, as it does not require costly appraisals. Of course, there are disadvantages to the mechanism as well. The tax authority does need to acquire the cargo in some cases, which implies costs of storage and resale, for example. Still, such costs are implicitly taken into account in the general model we propose. The required tax revenue that the mechanism allows the government to raise, are 
net of such costs.

We set up a general framework, that also allowed us to study other issues, such as the termination of a public-private partnership, and the auditing of income tax returns. In all cases, we assumed that one party is fully informed about the true value of some item, whereas the other party is not informed at all. In all cases, we showed that using a mechanism similar to that of the Sound Dues allows the uninformed party to obtain its required revenue in expected value. For the case of ending a partnership, a substantial body of literature has studied other and more complicated mechanisms in contexts where both parties are informed (for a survey, see Moldovanu, 2002). Yet, the beauty of the mechanism we described in this paper is that being completely uninformed does not hurt a party at all in acquiring the expected value of its share in the partnership.

\section{References}

Ash, R. B. (1972): Real Analysis and Probability. Academic Press, New York.

Chung, K.-S., and J. Ely (2007): "Foundations of Dominant-Strategy Mechanisms," Review of Economic Studies, 74(2), 447-476.

Gerchak, Y., And J. D. Fuller (1992): "Optimal Value Declaration in "Buy-Sell" Situations," Management Science, 38(1), 48-56.

Luce, R., And H. Raiffa (1957): Games and Decisions. Wiley, New York.

Maczak, A. (1972): Miedzy Gdanskiem a Sundem: Studia nad Handlem Baltyckim od Polowy XVI to Polowy XVII Wieku. Panstwowe Wydawnictwo Naukowe, Warsaw.

Moldovanu, B. (2002): "How to Dissolve a Partnership," Journal of Institutional and Theoretical Economics, 158, 66-80. 
Niou, E. M., And G. Tan (1994): "An Analysis of Dr. Sun Yat-sen's Self-assessment Scheme for Land Taxation," Public Choice, 78, 103-114.

Odlyzko, A. (2004): "The Evolution of Price Discrimination in Transportation and its Implications for the Internet," Review of Network Economics, 3, 323-346.

Shasha, D. E. (2007): "The Tolls of Elsinore," Scientific American, August, Available online at http://www.sciam.com/article.cfm?id=the-tollsof-elsinore.

Sun, Y. (1924): The Three Principles of the People. Translated into English by Frank W. Price. China Publishing Co., Taipei, Taiwan, ROC.

Wilson, R. (1987): "Game-Theoretic Approaches to Trading Processes," in Advances in Economic Theory, ed. by T. Bewley, Fifth World Congress, Econometric Society Monograph Series, No. 12, pp. 33-77. Cambridge University Press, Cambridge.

\section{Appendix}

Proof of Proposition 3 Observe that ex ante there is a joint distribution of $v$ and $m$. Nature chooses $v$ and then type $v$ employs a (possibly mixed) strategy resulting in a (random) $m$. Then the claimant observes $m$ and the conditional distribution of $v$ is recovered. Since, in general, the distributions of $v$ and $m$ may be degenerate, we need the following results from measure theory in the derivations below (for a comprehensive discussion of measure theory, see Ash, 1972). Below the subindex associated with an expectation symbol denotes the probability measures which are relevant while taking the expectation.

Lemma 1. Let $h_{1}: \mathbb{R} \rightarrow \mathbb{R}$ and $h_{2}: \mathbb{R} \rightarrow \mathbb{R}$ be continuously differentiable and bounded functions. Furthermore, let $X$ and $Y$ be two random variables with 
probability spaces $\left(\Omega_{X}, \mathcal{B}\left(\Omega_{X}\right), \mu_{X}\right)$ and $\left(\Omega_{Y}, \mathcal{B}\left(\Omega_{Y}\right), \mu_{Y}\right)$, which are respectively the outcome space, the associated Borel-field and a probability measure. Denote the combined probability space by $\left(\Omega_{X} \times \Omega_{Y}, \mathcal{B}\left(\Omega_{X}\right) \otimes \mathcal{B}\left(\Omega_{Y}\right), \mu_{X} \otimes \mu_{Y}\right)$. Then:

(i) $\mathbb{E}_{X Y}\left[h_{1}(x)+h_{2}(y)\right]=\mathbb{E}_{X}\left[h_{1}(x)\right]+\mathbb{E}_{Y}\left[h_{2}(y)\right]$

(ii) $\mathbb{E}_{Y}\left[\mathbb{E}_{X}\left[h_{1}(x) \mid y\right]\right]=\mathbb{E}_{X}\left[h_{1}(x)\right]$.

Proof. Ad (i) The expected value is given by:

$$
\begin{aligned}
\mathbb{E}_{X Y}\left[h_{1}(x)+h_{2}(y)\right] & =\int_{\Omega_{X} \times \Omega_{Y}}\left[h_{1}(x)+h_{2}(y)\right] \mathrm{d} \mu_{X} \otimes \mu_{Y} \\
& =\int_{\Omega_{X} \times \Omega_{Y}} h_{1}(x) \mathrm{d} \mu_{X} \otimes \mu_{Y}+\int_{\Omega_{X} \times \Omega_{Y}} h_{2}(y) \mathrm{d} \mu_{X} \otimes \mu_{Y} .
\end{aligned}
$$

Applying Fubini's Theorem (see Ash, 1972) yields:

$$
\begin{aligned}
\mathbb{E}_{X Y}\left[h_{1}(x)+h_{2}(y)\right] & =\int_{\Omega_{X}} \mathrm{~d} \mu_{X}(x) \int_{\Omega_{Y}} h_{1}(x) \mathrm{d} \mu_{Y}(y)+\int_{\Omega_{Y}} \mathrm{~d} \mu_{Y}(y) \int_{\Omega_{X}} h_{2}(y) \mathrm{d} \mu_{X}(x) \\
& =\int_{\Omega_{X}} h_{1}(x) \mathrm{d} \mu_{X}(x)+\int_{\Omega_{Y}} h_{2}(y) \mathrm{d} \mu_{Y}(y) \\
& =\mathbb{E}_{X}\left[h_{1}(x)\right]+\mathbb{E}_{Y}\left[h_{2}(y)\right] .
\end{aligned}
$$

Ad (ii) See Theorem 6.5.4 (a) of Ash (1972).

Let us now consider the linear case, where $R_{c}(v, m)=\alpha v+\beta m$ and $A_{c}(v, m)=\gamma v+\delta m$. Assumptions 2, 4 and 5 imply that

(a) either $\alpha>0>\gamma$ and $\beta<\delta$

(b) or $\gamma>0>\alpha$ and $\beta>\delta$.

Note that all functions we encounter are continuously differentiable and bounded. The expected payoff for the declarer in any signal-independent equilibrium is given by:

$$
\begin{aligned}
\mathbb{E}_{V M}\left[p R_{d}(v, m)+(1-p) A_{d}(v, m)\right] & =\frac{1}{1+c} \mathbb{E}_{V M}\left[c R_{d}(v, m)+A_{d}(v, m)\right] \\
& =\frac{1}{1+c} \mathbb{E}_{V}[S(v)],
\end{aligned}
$$


where the last equality follows from Lemma 1.

In a signal-independent equilibrium the claimant should be indifferent between Reject and Accept:

$$
\mathbb{E}_{V}\left[R_{c}(v, m)-A_{c}(v, m) \mid m\right]=\mathbb{E}_{V}[(\alpha-\gamma) v+(\beta-\delta) m \mid m]=0 .
$$

Rewriting this we see that

$$
\mathbb{E}_{V}[v \mid m]+\rho m=0
$$

where $\rho \equiv \frac{\beta-\delta}{\alpha-\gamma}<0$. Note that this equation only needs to hold for $m$ that are played in equilibrium.

The expected payoff for the claimant in a signal-independent equilibrium is:

$$
\mathbb{E}_{V M}\left[p R_{c}(v, m)+(1-p) A_{c}(v, m)\right]
$$

Note that this is an expectation over a bivariate distribution. Choosing $\lambda \equiv p \alpha+(1-p) \gamma$ and $\mu \equiv p \beta+(1-p) \delta$, we see that the expected payoff is:

$$
\begin{aligned}
\text { Payoff for the claimant } & =\mathbb{E}_{V M}[\lambda v+\mu m] \\
& =\lambda \mathbb{E}_{V}[v]+\mu \mathbb{E}_{M}[m] \\
& =\lambda \mathbb{E}_{V}[v]-\frac{\mu}{\rho} \mathbb{E}_{M}\left[\mathbb{E}_{V}[v \mid m]\right] \\
& =\left(\lambda-\frac{\mu}{\rho}\right) \mathbb{E}_{V}[v],
\end{aligned}
$$

where Lemma 1 is used repeatedly. 


\section{General model}

\begin{tabular}{rcc}
\multicolumn{1}{c}{} & Reject & Accept \\
\cline { 2 - 3 } Declarer & $R_{d}(v, m)$ & $A_{d}(v, m)$ \\
Claimant & $R_{c}(v, m)$ & $A_{c}(v, m)$
\end{tabular}

\section{Taxation}

\begin{tabular}{rcc}
\multicolumn{1}{c}{} & Purchase & Tax \\
\cline { 2 - 3 } Taxpayer & $-(v-m)$ & $-t m$ \\
Government & $v-m$ & $t m$
\end{tabular}

\section{Dissolving a public-private partnership}

\begin{tabular}{rcc}
\multicolumn{1}{c}{} & Sell (privatize) & Buy (nationalize) \\
\cline { 2 - 3 } Private party & $v-m$ & $m$ \\
Government & $m$ & $\delta(v, r)-m$
\end{tabular}

\section{Shotgun clauses}

\begin{tabular}{rcc} 
& Sell & Buy \\
\cline { 2 - 3 } Declarer & $(v-m) s$ & $-(v-m)(1-s)$ \\
Claimant & $-(v-m) s$ & $(v-m)(1-s)$
\end{tabular}

\section{Tax auditing}

\begin{tabular}{r|cc}
\multicolumn{1}{c}{ Not audit } & Audit \\
\cline { 2 - 3 } Manager & $-t v-f(v-m)$ & $-t m$ \\
Owner & $t v+f(v-m)-K$ & $t m$
\end{tabular}

\section{Allocating an indivisible item}

\begin{tabular}{ccc} 
& Item & Money \\
\cline { 2 - 3 } Player 1 & $-\frac{1}{2}(v-T)-m$ & $\frac{1}{2}(v-T)+m$ \\
Player 2 & $\frac{1}{2}(v-T)+m$ & $-\frac{1}{2}(v-T)-m$
\end{tabular}

Table 1: Payoffs for the general model and its applications. 